\title{
Development and Utilization of the Functional Co- dominant KASP Marker for Thermo-sensitive Genic Male Sterility in Rice
}

\section{Yongbin Qi ( $\sim$ qi_yongbin@hotmail.com )}

Zhejiang Academy of Agricultural Sciences

\section{LinYou Wang}

Institute of Crop Science and Nuclear Technology Utilization, Zhejiang Academy of Agricultral Sciences

Jian Song

Institute of Crop Science and Nuclear Technology Utilization, Zhejiang Academy of Agricultural Sciences

\section{Guohua Ma}

Sourthern Zhejiang Key Laboratory of Crop Breeding, Wenzhou Vocational College of Agriculture and Sciences

\section{Jianjun Wang}

Institute of Crop Science and Nuclear Technology Utilization, Zhejiang Academy of Agricultural Sciences

\section{Research Article}

Keywords: Two-line hybrid rice, Kompetitive Allele-Specific PCR, Thermo-sensitive genic male sterility, SNP, MAS

Posted Date: March 2nd, 2021

DOl: https://doi.org/10.21203/rs.3.rs-260801/v1

License: (c) (i) This work is licensed under a Creative Commons Attribution 4.0 International License. Read Full License

Version of Record: A version of this preprint was published at Genetic Resources and Crop Evolution on July 29th, 2021. See the published version at https://doi.org/10.1007/s10722-021-01249-7. 


\title{
Development and utilization of the functional co-dominant KASP marker for thermo-sensitive genic male sterility in rice
}

Yongbin Qi · Linyou Wang · Jian Song · Guohua Ma · Jianjun Wang

Y. Qi L. Wang · J. Song · J. Wang

Institute of Crop Science and Nuclear Technology Utilization, Zhejiang Academy of Agricultural Sciences, Hangzhou, Zhejiang, 310021, China.

G. Ma

Southern Zhejiang Key Laboratory of Crop Breeding, Wenzhou Vocational College of Agriculture and Science, Wenzhou, Zhejiang, 325006, China

e-mail: qi_yongbin@hotmail.com, Wangij4197@163.com

\begin{abstract}
Thermos-sensitive genic male sterility (TGMS) is the important genetic resource in two-line hybrid rice breeding. The pollen fertility of TGMS lines is regulated by a single point mutation on the TGMS genes. Based on the single nucleotide polymorphism (SNP) and indel, KASP markers were developed and utilized in rice molecular breeding due to high-through detection with low cost and save time. In this study, we convert the SNPs on TGMS genes including $p / t m s 12-1$ and $t m s 9-1$ gene to the functional co-dominant KASP marker and used in two-line hybrid rice breeding. We can differentiate the TGMS lines conferring p/tms $12-1$ or tms $9-1$ from another TGMS lines by KASP assay. Of them, the genotype of Pei'ai64S and Hua201S containing p/tms 12-1 is homozygous GG genotype with blue signal, and the genotype of HengnongS-1 containing tms $9-1$ is homozygous CC genotype with red signal. KASP assay for tms $9-1$ gene was proved that the genetic mode is fit to one recessive Mendalian trait. The test of seed purity was performed by KASP marker for the two-line hybrid varieties of Liangyoupeijiu and Hualiangyou1206, which is consistent with the previous dCAPS marker. Moreover, the tms 12-1 and tms9-1 gene were pyramided in the same genetic background and the new TGMS lines were generated. Therefore, the KASP marker for TGMS genes developed in this study can be widely used in two-line hybrid rice breeding. It will provide a visual convenient toolkit for breeders to select the target individual plant by the high-throughput detection in the different two-line rice breeding program.
\end{abstract}

Key words Two-line hybrid rice $\cdot$ Kompetitive Allele-Specific PCR $\cdot$ Thermo-sensitive genic male 
sterility $\cdot \mathrm{SNP} \cdot \mathrm{MAS}$

Rice is the staple crops as the major food resource that supplied for half world's population (Khush 2001, Yuan2004). Hybrid rice is planted in many counties due to the potential of the higher production than traditional inbred lines. Presently, hybrid rice was constituted as two systems including three-line and two-line (Cheng et al. 2007). Two-line hybrid rice has many advantages in comparison with the conventional three-line system such as the self-propagation of P/TGMS lines under low- temperature and short-photoperiod conditions, male parents with no need of restorer gene in hybrid rice system, eliminating the adverse cytoplasmic effect on agronomic traits for the hybrid variety and so on (Yuan 1990; Virmani et al. 2003; Si et al. 2011). The photoperiod- and thermo-sensitive genic male sterile gene as the key factors that regulated the fertility conversion of P/TGMS lines under different environmental conditions plan a crucial role in the two-line hybrid rice. Since the past few years, many two-line hybrid rice varieties were bred and widely applied in grain production in China with the finding and development of P/TGMS lines (Si et al. 2011; Mou 2016).

To date, a lot of P/TGMS genes as major locus conferring P/TGMS trait were mapped and indentified into different Chromosome: these include TGMS genes tms 1, tms 2, tms3, tms4, tms5, tms6, tms6(t), tms9, tms9-1, tms in SA2, tmsX, ptgms2-1 and PGMS genes pms1, pms2, pms3 and p/tms12-1 (Fan and Zhang 2016). Further study showed that TMS9, TMSX, ptgms2-1 were mapped in the region of TMS 5 or the vicinity of TMS5 suggesting that tms 5 , tms $9, \operatorname{tms} X$ and ptgms $2-1$ are allelic mutants (Fan and Zhang 2016). Nongken58S, the first PGMS lines, were found in a conventional japonica inbred lines Nongken58 and its PGMS traits were mainly regulated by pms3 and p/tms12-1(Ding et al. 2012; Zhou et al. 2012; Zhu and Deng 2012). Pei'ai64S was a derived line bred from Nongken58S, but showed a TGMS trait in indica genetic background. A key single base substitution of C- to G- in p/tms 12-1 which resulted into a loss function of a small RNA named as osa-smR5864w was found in Pei'ai64S (Zhou et al. 2012). Tms5 encoded an RNase $Z^{\mathrm{S} 1}$ protein processing $U b_{L 40}$ mRNA was found from an early indica TGMS lines Annong S-1. A nonsense mutation C-to-A mutation at TMS5 gene creating a premature stop codon was the key factor that controls the TGMS trait in AnnongS-1 and its derived lines (Zhou et al. 2014). Different with p/tms12-1 and tms5, tms9-1 mapped on the rice Chromosome 9 is other TGMS gene found in an early indica TGMS lines HengnongS-1. A SNP of C to T at the candidate gene $O s M S 1$ resulting an amino acid substitution regulated the TGMS trait of 
Hengnong S-1(Qi et al. 2014). Interestingly, the SNP polymorphism on the different TGMS gene including $p / t m s 12-1, t m s 5$ and tms $9-1$ is the key factors to control the TGMS trait in TGMS lines and its derived lines. The different thermo-sensitive genic male sterility were determined by those SNP polymorphism, we can convert the SNPs to functional markers (FMs) and used in the mark-assisted breeding program in two-line hybrid rice system.

SNPs and Indel are the common mutations in genes that determine many important agronomic traits in rice breeding. The polymorphism of Indel can be identified as co-dominant marker due to the length of PCR production. With respect to genotyping for SNP, various methods have been developed, such as AS-PCR (allele specific PCR), CAPS (cleaved amplified polymorphism sequence), dCAPS (drived CAPS), HRM (high-resolution melting), TS-PCR (temperature switch PCR) are used in rice genotyping study. It is difficult to distinguish the heterozygotes from homozygotes by AS-PCR, as AS-PCR provides a dominant marker results (Gaudet et al. 2009). CAPS and dCAPS rely on the site-specific digestion of PCR products, so it is expensive and time-consuming and difficult to use in a large scale genotyping due to the low efficiency of the enzyme digestion (Chen et al. 2009). HRM is based on the melting curves analysis for different melting temperature to detect SNP alleles, it is need a set of complicated and multiround PCR and easy to lead to false positive (Yang et al. 2019). TS-PCR (Temperature Switch PCR) consists of a biphasic PCR with two sets of precisely designed primers. The target sequences enriched by a pair of site-specific primers at the first stage and then DNA containing the SNP allele were amplified and detected by the nested primers at the second stage, so it is time-consuming and laborious (Thanh and Khoo, 2014). With the identification and discovery of large amount SNP on genes that contribute to agronomic traits in rice, the technology for SNP genotyping will provide useful information to breeder for the selection of the parent combination with the desirable trait. KASP (Kompetitive Allele-Specific PCR) is a single-step genotyping technology that is based on terminal fluorescence reading similar to Taqman detection (Patil et al. 2017). Both methods require two fluorescent signals of the end-point by laser scanning to detect the individual samples with different genotypes; different fluorescent signals represent different SNP on the same locus. KASP marker has been used in a wide range of applications, such as germplasm identification, genotyping for SNP, bulked segregant analysis, and MAS, as it has many advantages with high throughput, highly accurate, good genetic stability, high flexibility, locus specificity and low cost (Semagn et al. 2014; Mackay et al. 2014; Cabral et al. 2014; Leal-Bertioli et al. 2015; Wu et al. 2017; Yang et al. 2019; Li et al. 2020; 
Makhoul et al.2020). Functional SNPs and Indels can directly determine the phenotypic trait, and such polymorphism can be developed into functional markers (FMs). Due to the high efficiency and low cost, conversion of those FMs into KASP markers will provide a convenient toolkit for breeder to select the target individual plant from different combinations population.

In this study, SNPs in the two thermo-sensitive genic male sterility genes ( $p /$ tms $12-1$ and tms $9-1)$ was converted into KASP marker and validated into MAS in two-line hybrid rice breeding. The new developed KASP markers can be used to distinguish different PTGMS lines, select the individual plants from different combination progeny, and test the seed purity of two-line hybrid varieties and pyramid two TGMS genes into the same genetic background. KASP assay provide a new reliable alternative method for the high throughput detection in two-line hybrid rice breeding.

\section{Materials and Methods}

Plant materials and fertility identification

Eighteen TGMS lines that were widely used in two-line hybrid rice production from the South of China were selected to test the KASP assay for TGMS genes. According to the previous reports and the source of TGMS genes, the TGMS trait of Hua201S and Pei'ai64S were regulated by $p /$ tms $12-1$ gene, and that of HengnongS-1were regulated by tms $9-1$ gene, others were regulated by tms 5 gene (Table 1 ) (Zhang et al. 2014; Zhang et al. 2015). In order to convert the SNPs in tms9-1 and p/tms12-1 genes, two sets of KASP markers were designed according to SNP allele (Table 2). To test the validation of KASP assay for tms9-1 gene, one cross combination with HengnongS-1×Minghui63 was used to detect the SNP allele of tms $9-1$ gene in $\mathrm{F}_{2}$ population. A total of 164 individual plants from the $\mathrm{F}_{2}$ population were selected and performed the KASP assay. For the validation of KASP assay for $p / t m s 12-1$, two hundred seeds of hybrid rice varieties with Liangyoupeijiu and Hualiangyou1206 were selected to test the seed purity respectively. Liangyoupeijiu and Hualiangyou1206 were bred with Pei'ai64S and Hua201S as female parent conferring $p / t m s 12-1$ gene, respectively. In order to pyramid $p / t m s 12-1$ and tms $9-1$ into the same genetic background, one combination cross with HengnongS- $1 \times$ Hua201 was prepared under the natural low temperature in Hainan Island, China at the winter. Individual plants with TGMS trait were selected from the $F_{2}$ population and generated $F_{3}$ and $F_{4}$ by self-propagation under 
low-temperature environmental condition. A total of 92 TGMS individual plants from this $\mathrm{F}_{4}$ population were selected to detect the p/tms $12-1$ and tms $9-1$ gene by KASP assay. All individual plants were grown at the end of May in the experimental farm of Zhejiang Academy of Agricultural Sciences, Hangzhou, China. Pollen of individual plant were collected from the anther at the stage of pre-flowering and stained with $1 \% \mathrm{I}_{2}$-KI solution. The fertility of pollen was observed under a NIKON ECLIPSE E100 light microscope. The plants which the pollens of them were unstained and irregular were classified as sterile plant; however the plants which the pollens of them were round and darkly stained were classified as fertile plant (Fig.1) (Qi et al. 2017).

DNA isolation and KASP SNP assay

At the rice heading stage, genomic DNA were extracted from the plant top second leaf with the length of 1-2 cm using the CTAB buffer according to the pollen fertility to avoid sampling mistake. The concentration of DNA were measured by a Nanodrop one ${ }^{c}-1000$ spectrophotometer (Thermo Fisher Scientific, USA) and adjusted into a working concentration with 100ng/L. For the marker development of KASP assay in this study, different SNP allele at the same locus was analyzed from the previous described report (Zhou et al. 2012; Qi et al. 2014). To design KASP marker for genotyping, about 200bp sequence surrounding the SNP locus on upstream and downstream were analyzed and the KASP primers were designed by the standard KASP guidelines and ordered from LGC Limited, UK. The standard FAM (5'-GAAGGTGACCAAGTTCATGCT -3') and HEX (5'

-GAAGGTCGGAGTCAACGGATT-3') were used and linked with the allele-specific primer which targeted with the SNP at the 3'end point. A common reverse primer was designed by the Primer 3 (Untergasser et al. 2012) with the product length of less than 100bp. For genotyping assay with KASP, genomic DNA was transfer into 374 well plate and dried at $65^{\circ} \mathrm{C}$ for $30 \mathrm{~min} .1 \mu 1$ KASP assay mixture comprised $0.5 \mu \mathrm{l} 2 \times \mathrm{KASP}$ master mixture (Standard Rox, LGC), $0.014 \mu \mathrm{l}$ each primer mix and $0.486 \mu \mathrm{l}$ $\mathrm{H}_{2} \mathrm{O} .1 \mu \mathrm{l}$ reaction mixture was added into each one sample. The PCR reaction was performed on the Hydrocycler-16 machine (LGC Limited, UK) using the following program: hot start at $94{ }^{\circ} \mathrm{C}$ for 15 min, followed by 10 touchdown cycles $\left(94{ }^{\circ} \mathrm{C}\right.$ for $20 \mathrm{~s}$; touchdown at $65^{\circ} \mathrm{C}$ initially and decreasing by $-1{ }^{\circ} \mathrm{C}$ per cycle for $\left.25 \mathrm{~s}\right)$, and then followed by 26 cycles of amplification $\left(94^{\circ} \mathrm{C}\right.$ for $20 \mathrm{~s}, 55^{\circ} \mathrm{C}$ for $\left.60 \mathrm{~s}\right)$. The temperature of PCR mixture was declined into room temperature, the read for fluorescence signal 
was performed at the Pherastar scanning machine (LGC Limited, UK). Once there have no clear results, an additional 3 cycles of amplification $\left(94^{\circ} \mathrm{C}\right.$ for $20 \mathrm{~s}, 55^{\circ} \mathrm{C}$ for $\left.60 \mathrm{~s}\right)$ can be added to further amplify. All KASP assays were applied at the Shanghai Baygene Biotechnologies Limited Company, Shanghai, China. Genotyping data were collected and viewed by SNP viewer 2 (LGC Limited, UK).

\section{Results}

Identification of TGMS lines and genetic analysis for tms9-1 gene by KASP assay

According to previous study, the $\mathrm{G} / \mathrm{T}$ and $\mathrm{C} / \mathrm{T}$ polymorphism located at the noncoding small RNA and the third exon of OsMS1 gene, which is responsible for the regulation of the trait of TGMS by p/tms 12-1 and tms9-1 gene, respectively (Zhou et al. 2012; Qi et al. 2014). Those functional SNPs can discriminate different genotypes such as $\mathrm{G} / \mathrm{G}, \mathrm{G} / \mathrm{C}$, and $\mathrm{C} / \mathrm{C}$ on $\mathrm{p} /$ tms $12-1$ gene and $\mathrm{C} / \mathrm{C}, \mathrm{C} / \mathrm{T}$ and $\mathrm{T} / \mathrm{T}$ on tms9-1 gene. KASP assay showed that two TGMS lines (Hua201S and Pei'ai64S) were classified as homozygous G/G genotypes, and other sixteen TGMS lines were determined to have homozygous C/C genotype on p/tms12-1 gene. One TGMS line (HengnongS-1) was classified as homozygous C/C genotype, but others were determined to have homozygous T/T genotype on $t m s 9-1$ gene (Fig 2). In order to validate the KASP marker on tms $9-1$ gene, one cross combination with HengnongS-1 $\times$ Minghui63 was prepared, and 164 individual plants from this $\mathrm{F}_{2}$ population were selected to genotype analysis for $t m s 9-1$ gene by the KASP assay. The results showed that 31 individual plants were classified as homozygous $\mathrm{C} / \mathrm{C}$ genotype which represented as the TGMS trait, 82 individual plants were classified as heterozygous $\mathrm{C} / \mathrm{T}$ genotype and 51 individual plants were classified as homozygous T/T genotype which all represented as non-TGMS trait (Fig 3). Chi squared analyses for genotype in the $\mathrm{F}_{2}$ population showed that the segregation ratio of three genotypes $(\mathrm{C} / \mathrm{C}, \mathrm{C} / \mathrm{T}, \mathrm{T} / \mathrm{T})$ in the $\mathrm{F}_{2}$ populations was at $1: 2.65: 1.65\left(\chi_{0.05}^{2}=4.89<5.99, d f=2\right)$, which fitted to $1: 2: 1$, indicating that the genetic model of tms $9-1$ was a simple recessive Mendalian trait that is consistent with our previous study (Qi et al. 2014).

KASP assay for the seed purity of two-line hybrid rice varieties 
To validate the KASP assay used in the two-line hybrid rice varieties, two two-line hybrid rice varieties including Liangyoupeijiu and Hualiangyou1206 which were widely planted in the south of China were selected to measure the seed purity of the variety. $200 \mathrm{~F}_{1}$ individual plants seeds of them were randomly selected to detect the seed purity by the KASP marker. The KASP assay showed that the genotype of 196 individual plant of Liangyoupeijiu was heterozygous C/G genotype with green signal, and three plants was homozygous G/G genotype with blue signal same with female parent, but only one plant was homozygous $\mathrm{C} / \mathrm{C}$ genotype with red signal same with male parent or other conventional plants (Fig 4). The pollen fertility of individual plant showed that the plants with blue signal were male sterile, whereas the plants with green signal and red signal were male fertile. The KASP assay also showed that the genotype of all $200 \mathrm{~F}_{1}$ plants of Hualiangyou1206 was heterozygous $\mathrm{C} / \mathrm{G}$ genotype with green signal (Fig 4), which is consistent with our previous study tested by one dCAPS marker (Qi et al. 2017).

Pyramiding of tms $9-1$ and $p / t m s 12-1$ gene by KASP assay

To generate the new TGMS lines with different TGMS genes including tms $9-1$ and $\mathrm{p} / \mathrm{tms} 12-1$, a total of $92 \mathrm{~F}_{4}$ individual plants with TGMS trait from one combination cross with HengnongS- $1 \times$ Hua201S were prepared to detect TGMS gene by KASP marker. KASP assay for $t m s 9-1$ gene showed that the genotype of 77 individual plants was homozygous C/C genotype with red signal same with Hengnongs-1, and that of 13 individual plants was heterozygous C/T genotype with green signal, but two individual plants cannot be detected. KASP assay for $p / t m s 12-1$ gene showed that the genotype of 76 individual plants was homozygous G/G genotype with blue signal same with Hua201S, and that of 15 individual plants was heterozygous $\mathrm{C} / \mathrm{G}$ genotype with green signal, but only one individual plant cannot be detected. So, the genotype of 74 individual plants was homozygous $\mathrm{C} / \mathrm{C}$ for $t m s 9-1$ gene and G/G for $p / t m s 12-1$ gene (Fig 5). Different TGMS genes were pyramided into the same genetic background by MAS with KASP assay.

\section{Discussion}

SNPs are one-base variation in a single nucleotide allele that is widely distributed on the different rice 
accessions with high density of genetic variants which can occur in coding and non-coding regions. With the development of next-generation sequencing (NGS) technology, a large scale of SNPs were generated in diverse rice genome (Kumar et al. 2012). As the functional SNPs can directly reflect natural genetic variability of rice accession, a high-throughput genotyping technology for SNPs is need, and it will facilitates breeders to select the parents for the improvement of desirable traits.

Pariasca-Tanaka et al (2015) reported that a total of 2,015 KASP markers were found and 1,890 existing KASP can be applicable to indica rice. Some SNPs and Indel that determine the rice eating and cooking quality have been converted into KASP markers and used to improve the rice quality by the molecular marker assisted selection (MAS) combined with conventional breeding approach (Yang et al.2019).

Two-line hybrid rice is widely planted in the south of China due to its higher yield and other advantages than three-line hybrid rice (Si et al. 2012; Qi et al. 2014). Thermo sensitive genic male sterile line is the most important genetic resource in two-line hybrid rice breeding, and the conversion of its pollen fertility were regulated by TGMS genes under different environmental conditions. To date, many TGMS genes were identified and cloned on different rice Chromosome, but only tms 5, p/tms 12-1 and tms9-1 genes have been used in the two-line hybrid breeding in China (Zhou et al. 2014; Zhou et al.2012; Qi et al. 2014). The pollen fertility of different TGMS lines was regulated by the SNPs on the TGMS genes. In the TGMS line including Pei'ai64S and Hua201S, a point mutation from G to C on the lncRNAs of $p / t m s 12-1$ gene controls the pollen fertility under different environmental conditions. Meanwhile, a point mutation from $\mathrm{C}$ to $\mathrm{T}$ on the third exon of tms $9-1$ gene controls the conversion of pollen fertility in HengnongS-1 line (Zhou et al.2012; Qi et al. 2014). In this study, we successfully convert the SNPs on p/tms 12-1 and tms9-1 to KASP marker and used in two-line hybrid rice breeding. These functional co-dominant KASP assay can be used to distinguish different TGMS lines conferring the TGMS genes such as $p / t m s 12-1$ or tms $9-1$ through different fluorescent signal. These visual markers can also be used to analyze the genetic analysis for tms $9-1$ gene and detect the seed purity of two-line hybrid rice varieties in our study. The results of genetic analysis for tms $9-1$ gene is consistent with our previous study (Qi et al. 2014), which indicated that the KASP assay for tms9-1 gene will be validated to make use in two-line hybrid breeding by MAS. The test of seed purity for two-line hybrid rice varieties is also consistent with our previous study in which a co-dominant dCAPS marker was used (Qi et al. 2017). However, it is little difficult and high cost for high-throughput genotyping and 
detection by dCASP marker. So, the KASP marker has the distinct advantages compared to the previously published marker especially in large scale detection (Zhang et al. 2014; Qi et al. 2017). It will facilitate breeder to precisely identify the genotype of different TGMS genes with low cost and save time in two-line hybrid rice breeding combined with conventional breeding. In our study, we use the KASP marker to pyramid tms $9-1$ and $p / t m s 12-1$ into the same genetic background and generate the new TGMS lines in which the TGMS trait of them was regulated by tms $9-1$ and $p / t m s 12-1$. Utilization of KASP assay for $p / t m s 12-1$ and $t m s 9-1$ gene will overcome the difficulty that we cannot identify the TGMS genes in the p/tms12-1/tms9-1 TGMS lines by the regular phenotype analysis for pollen fertility.

Acknowledgements Financial support was provided for this research partly by the Project of the Science and Technology Department of Zhejiang Province, China (Grant number LGN21C130005, 2016C02050), the Project of the Molecular Designed Japonica Breeding with High Quality and Yield in the Middle-Low Reaches of Yangzi River (Grant Number 2017YFD0100302) and the Talent Training Project of Zhejiang Academy of Agricultural Sciences (Grant Number 2018R16R08E01).

Authors' contribution YQ and JW conceived and designed the study. YQ, LW, JS and GM performed the study test. YQ, LW and JS contributed the KASP assay. YQ and GM prepared the materials and populations. YQ and JW wrote the manuscript.

Ethical Statement The authors declare that they have no conflict of interest. This study complied with the ethical standards of China, where this research was carried out.

\section{References:}

Cabral AL, Jordan MC, McCartney CA, You FM, Humphreys DG, MacLachlan R, Pozniak CJ (2014) Identification of candidate genes, regions and markers for pre-harvest sprouting resistance in wheat (Triticum aestivum L.) BMC Plant Biol 14(1):340. https:/ /doi.org/10.1186/s12870-014-0340-1

Chen T, Zhang Y, Zhao L, Zhu Z, Lin J, Zhang S, Wang C (2009) A cleaved amplified polymorphic sequence marker to detect variation in Wx locus conditioning translucent endosperm in Rice. Rice Sci 16(2):106-110. https:/ /doi.org/10.1016/S1672-6308 (08)60065-7

Cheng SH, Zhuang JY, Fan YY, Du JH, Cao LY (2007) Progress in research and development on hybrid rice: a super-domesticate in China. Ann Bot (Lond) 100(5):959-966.

Ding JH, Lu Q, Ouyang YD, Mao HL, Zhang PB, Yao JL, Xu CG, Li XH, Xiao JH, Zhang QF (2012) A long 
noncoding RNA regulates photoperiod-sensitive male sterility, an essential component of hybrid rice. Proc Natl Acad Sci USA 109(7):2654-2659. https:/ /doi.org/10.1073/pnas.1121374109

Fan Y, Zhang Q (2018) Genetic and molecular characterization of photoperiod and thermo-sensitive male sterility in rice. Plant Reprod 31(1):3-14. 10. https:/ /doi.org/1007/s00497-017-0310-5

Gaudet M, Fara A-G, Beritognolo I, Sabatti M (2009) Allele-specific PCR in SNP genotyping. Method Mol Biol 578:415-424. https://doi.org/10.1007/978-1-60327-411-1_26

Leal-Bertioli SCM, Cavalcante U, Gouvea EG, Ballén-Taborda C, Shirasawa K, Guimarães PM, Jackson SA, Moretzsohn MC (2015) Identification of QTLs for rust resistance in the peanut wild species Arachis magna and the development of KASP markers for marker-assisted selection. G3 Genes Genomes Genetics 5(7):1403-1413. https://doi.org/ 10.1534/g3.115.018796.

Mackay IJ, Bansept-Basler P, Barber T, Bentley AR, Cockram J, Gosman N, Greenland AJ, Horsnell R, Howells R, O’Sullivan DM, Rose GA (2014) An eight-parent multiparent advanced generation inter-cross population for winter-sown wheat: creation, properties, and validation. G3 Genes Genomes Genetics 4(9):1603-1610. https://doi.org/ 10.1534/g3.114.012963

Khush GS (2001) Green revolution: the way forward. Nat Rev Genet 2(10):815-822. https://doi.org/10.1038/35093585

Kumar S, Banks TW, Cloutier S. SNP Discovery through Next-Generation Sequencing and Its Applications. Int J Plant Genomics. 2012; 2012:831460. https://doi.org/10.1155/2012/831460

Li Z, Yu H, Li X, Zhang B, Ren W, Liu X, Fang Z, Yang L, Zhuang M, Lv H, Zhang Y (2020) Kompetitive allele-specific PCR (KASP) genotyping and heterotic group classification of 244 inbred lines in cabbage (Brassica oleracea L. var. capitata). Euphytica 216:106. https://doi.org/10.1007/s10681-020-02640-8

Makhoul M, Rambla C, Voss-Fels KP, Hickey LT, Snowdon RJ, Obermeier C(2020) Overcoming polyploidy pitfalls: a user guide for effective SNP conversion into KASP markers in wheat. Theor Appl Genet 133(8):2413-2430. https://doi.org/10.1007/s00122-020-03608-x

Mou T (2016) The research progress and prospects of two-line hybrid rice in China. Chinese Sci Bull 61:3761-3769.

Pariasca-Tanaka J, Lorieux M, He C, McCouch S, Thomson MJ, WissuwaM(2015) Development of a SNP genotyping panel for detecting polymorphisms in Oryza glaberrima/O. sativa interspecific crosses. Euphytica 201(1):67-78. https://doi.org/10.1007/s10681-014-1183-4.

Patil G, Chaudhary J, Vuong TD, Jenkins B, Qiu D, Kadam S, Shannon GJ, Nguyen HT (2017) Development of 
SNP genotyping assays for seed composition traits in soybean. Int J Plant Genomics 2017:6572969. https://doi.org/10.1155/2017/6572969

Pham A-T, Harris DK, Buck J, Hoskins A, Serrano J, Abdel-Haleem H, Cregan P, Song Q, Boerma HR, Li Z (2015) Fine mapping and characterization of candidate genes that control resistance to Cercospora sojina K. Hara in two soybean germplasm accessions. PLoS One 10(5):e0126753. https://doi.org/10.1371/journal.pone.0126753

Qi Y, Liu Q, Zhang L, Mao B, Yan D, Jin Q, He Z (2014) Fine mapping and candidate gene analysis of the novel thermo-sensitive genic male sterility tms9-1 gene in rice. Theor Appl Genet 127:1173-1182. https://doi.org/10.1007/s00122-014-2289-8

Qi Y, Wang L, Gui J, Zhang L, Liu Q, Wang J (2017) Development and validation of a functional co-dominant SNP marker for the photoperiod thermo-sensitive genic male sterility pms3 (p/tms12-1) gene in rice. Breed Sci 67(5):535-539. https://doi.org/10.1270/jsbbs.16138

Semagn K, Babu R, Hearne S, Olsen M (2014) Single nucleotide polymorphism genotyping using kompetitive allele specific PCR (KASP): overview of the technology and its application in crop improvement. Mol Breed 33:1-14. https://doi.org/10.1007/s11032-013-9917-x

Si HM, Liu WZ, Fu YP, Sun ZX, Hu GC (2011) Current situation and suggestions for development of two-line hybrid rice in China. Chin J Rice Sci 25(5):544-552.

Thanh le P, Khoo K (2014) Temperature switch PCR (TSP): a gel-based molecular marker technique for investigating single nucleotide polymorphisms. Methods Mol Biol 1145:37-46. https://doi.org/10.1007/978-1-4939-0446-4_3

Untergasser A, Cutcutache I, Koressaar T, Ye J, Faircloth BC, Remm M, Rozen SG (2012) Primer3-new capabilities and interfaces. Nucleic Acids Res 40(15):e115. https://10.1093/nar/gks596

Virmani S, Sun Z, Mou T, Jauhar AA, Mao C (2003) Two-line hybrid rice breeding manual. International Rice Research Institute, Los Baños, p 88.

Wu JH, Wang QL, Kang ZS, Liu SJ, Li HY, Mu JM, Dai MF, Han DJ, Zeng QD, Chen XM(2017) Development and validation of KASP-SNP markers for QTL underlying resistance to stripe rust in common wheat cultivar P10057. Plant Dis 101:2079-2087. https://doi.org/ 10.1094/PDIS-04-17-0468-RE

Yang G, Chen S, Chen L, Sun K, Huang C, Zhou D, Huang Y, Wang J, Liu Y, Wang H, Chen Z, Guo T (2019) Development of a core SNP arrays based on the KASP method for molecular breeding of rice. Rice 12(1):21. https://doi.org/10.1186/s12284-019-0272-3 
Yang G, Chen S, Chen L, Gao W, Huang Y, Huang C, Zhou D, Wang J, Liu Y, Huang M, Xiao W, Wang H, Guo T, Chen Z(2019) Development and utilization of functional KASP markers to improve rice eating and cooking quality through MAS breeding. Euphytica 215, 66. https://doi.org/10.1007/s10681-019-2392-7

Yuan LP (2004) Hybrid rice technology for food security in the world. Crop Res 18:185-186. https://doi.org/10.16848/j.cnki.issn.1001-5280.2004.04.001

Yuan LP (1990) Progress of two-line system hybrid rice breeding. Sci Agric Sin 23(3):1-6.

Zhang H, Huang J, Liu Q, Nawaz, Lu H, Gong J, Zhu Y, Yan W, Shu Q (2014) Characterization of an RNase Z nonsense mutation identified exclusively in environment-conditioned genic male sterile rice. Mol Breeding 34, 481-489. https://doi.org/10.1007/s11032-014-0051-1

Zhang H, Chen X, Huang J, E Z, Gong J, Shu Q(2015)Identification and Transition Analysis of Photo/Thermo-Sensitive Genic Male Sterile Genes in Two-Line Hybrid Rice in China. Scientia Agricultura Sinica 48(1): $1-9$.

Zhou H, Liu QJ, Li J, Jiang DG, Zhou LY, Wu P, Lu S, Li F, Zhu LY, Liu ZL, Chen LT, Liu YG, Zhuang CX (2012) Photoperiod- and thermo-sensitive genic male sterility in rice are caused by a point mutation in a novel noncoding RNA that produces a small RNA. Cell Res 22(4):649-660. https://doi.org/10.1038/cr.2012.28

Zhou H, Zhou H, Zhou M, Yang Y, Li J, Zhu L, Jiang D, Dong J, Liu Q, Gu L, Zhou L, Feng M, Qin P, Hu X, Song C, Shi J, Song X, Ni E, Wu X, Deng Q, Liu Z, Chen M, Liu YG, Cao X, Zhuang C (2014) RNase Z(S1) processes UbL40 mRNAs and controls thermosensitive genic male sterility in rice. Nat Commun 5:4884-4892. https://doi.org/10.1038/ncomms5884

Zhu D, Deng X W (2012) A non-coding RNA locus mediates environment-conditioned male sterility in rice. Cell Res 22: 791-792. https://doi.org/10.1038/cr.2012.43 


\section{Figure legends}

Fig. 1 Pollen fertility of rice under high temperature and long photo-period environmental conditions a: unstained and irregular pollen was classified as sterile plant; b: round and darkly stained was classified as fertile plant.

Fig. 2 Eighteen TGMS lines were performed by KASP assay for $p / t m s 12-1$ and tms $9-1$ gene

a: KASP assay of the TGMS lines for p/tms12-1 gene, Hua201S and Pei'ai64S were homozygous G/G genotype with blue signal; and other TGMS lines were homozygous C/C genotype with red signal. b: KASP assay for the TGMS lines with KASP assay for tms9-1 gene, HengnongS-1 were homozygous C/C genotype with red signal; and other TGMS lines were homozygous T/T genotype with blue signal.

Fig. 3 KASP assay of individual plant for $t m s 9-1$ gene in partial $F_{2}$ population

Note: The genotype of individual plant was homozygous $\mathrm{C} / \mathrm{C}$ with red signal which represented as TGMS trait; the genotype of individual plant was heterozygous $\mathrm{C} / \mathrm{T}$ with green signal and homozygous $\mathrm{T} / \mathrm{T}$ with blue signal which represented as non-TGMS trait.

Fig. 4 Test of seed purity for two-line hybrid rice varieties by KASP assay

a: KASP assay for Liangyoupeijiu, the genotype of most part of individual plants is heterozygous $\mathrm{C} / \mathrm{G}$ with green signal, and the genotype of three individual plants is homozygous $\mathrm{G} / \mathrm{G}$ with blue signal same with female parent Pei'ai64S, and the genotype of one individual plant is homozygous $\mathrm{C} / \mathrm{C}$ with red signal same with male parent or other conventional plants.

b: KASP assay for Hualiangyou1206, the genotype of all individual plants is heterozygous C/G with green signal. Fig. 5 pyramiding of tms $9-1$ and $p / t m s 12-1$ gene by KASP assay

a: KASP assay for tms $9-1$ gene in $\mathrm{F}_{4}$ population.

b: KASP assay for $p / t m s 12-1$ gene in $\mathrm{F}_{4}$ population.

Note: the individual plants with homozygous $\mathrm{G} / \mathrm{G}$ and $\mathrm{C} / \mathrm{C}$ were selected as the TGMS plant pyramiding of $t m s 9-1$ and $p / t m s 12-1$ gene. 


\section{Figures}
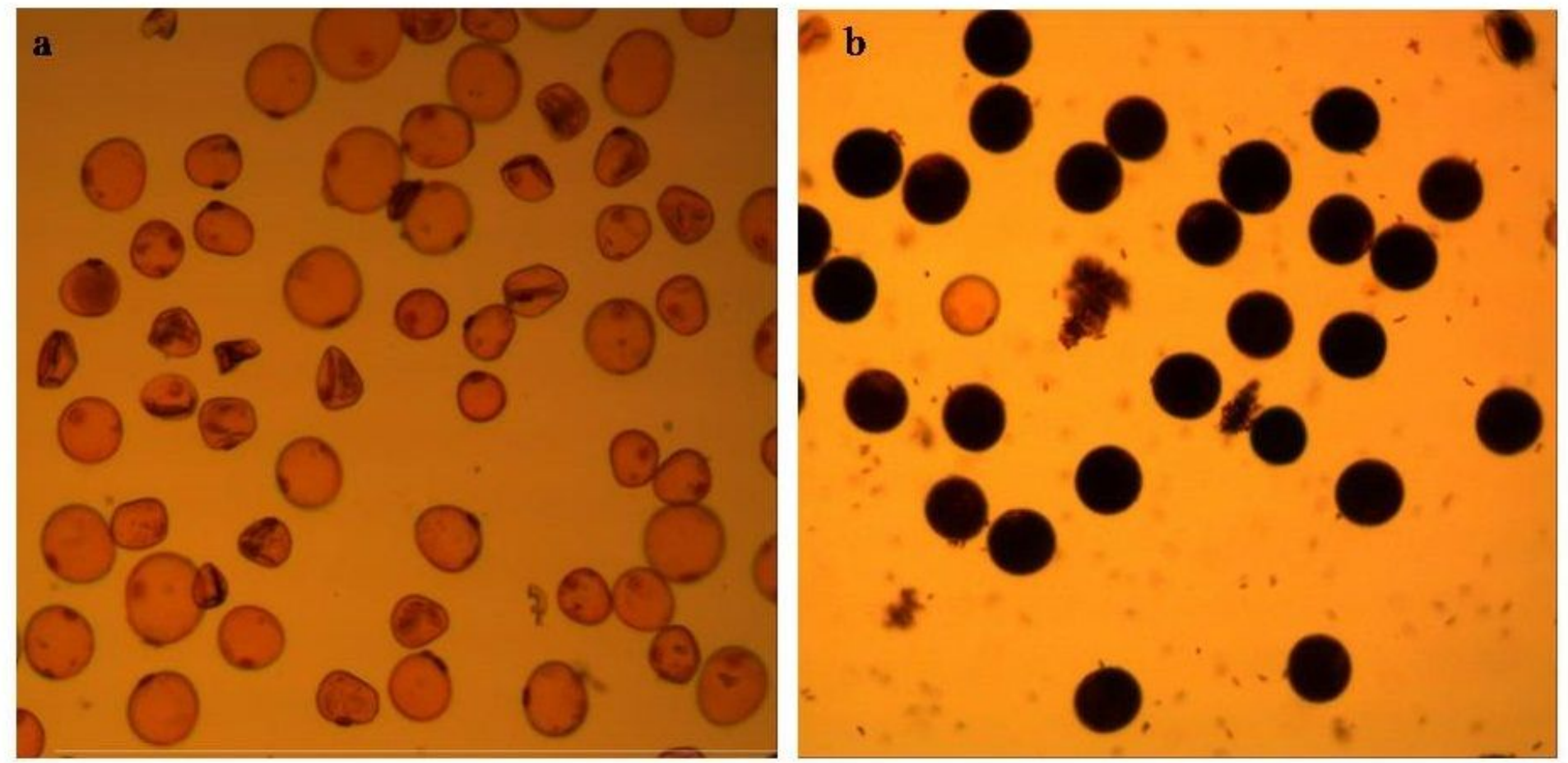

\section{Figure 1}

Pollen fertility of rice under high temperature and long photo-period environmental conditions a: unstained and irregular pollen was classified as sterile plant; b: round and darkly stained was classified as fertile plant.
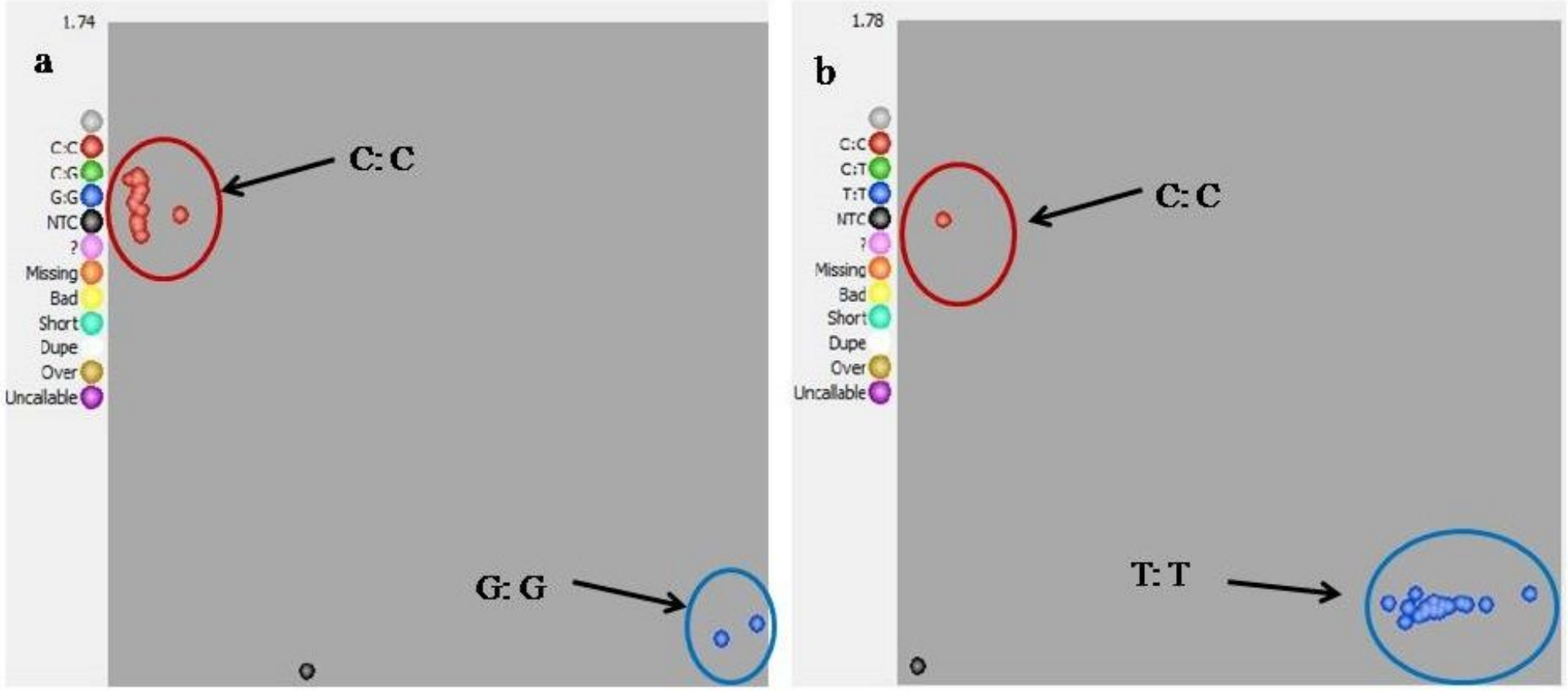

Figure 2 
Eighteen TGMS lines were performed by KASP assay for $\mathrm{p} / \mathrm{tms} 12-1$ and tms9-1 gene a: KASP assay of the TGMS lines for p/tms12-1 gene, Hua201S and Pei'ai64S were homozygous G/G genotype with blue signal; and other TGMS lines were homozygous $\mathrm{C} / \mathrm{C}$ genotype with red signal. b: KASP assay for the TGMS lines with KASP assay for tms9-1 gene, HengnongS- 1 were homozygous $\mathrm{C} / \mathrm{C}$ genotype with red signal; and other TGMS lines were homozygous T/T genotype with blue signal.

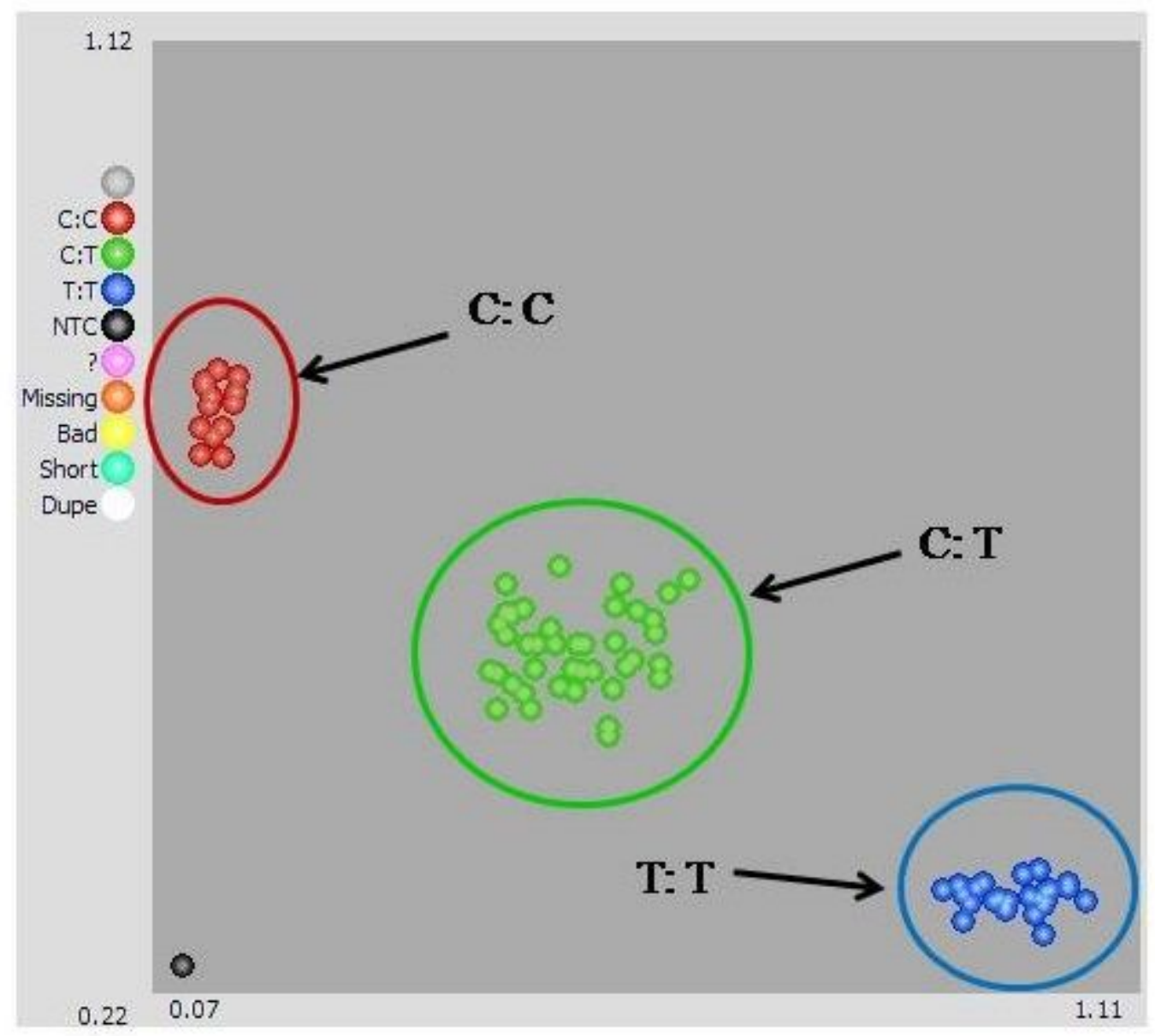

\section{Figure 3}

KASP assay of individual plant for tms9-1 gene in partial F2 population Note: The genotype of individual plant was homozygous $\mathrm{C} / \mathrm{C}$ with red signal which represented as TGMS trait; the genotype of individual plant was heterozygous $\mathrm{C} / \mathrm{T}$ with green signal and homozygous $\mathrm{T} / \mathrm{T}$ with blue signal which represented as non-TGMS trait. 

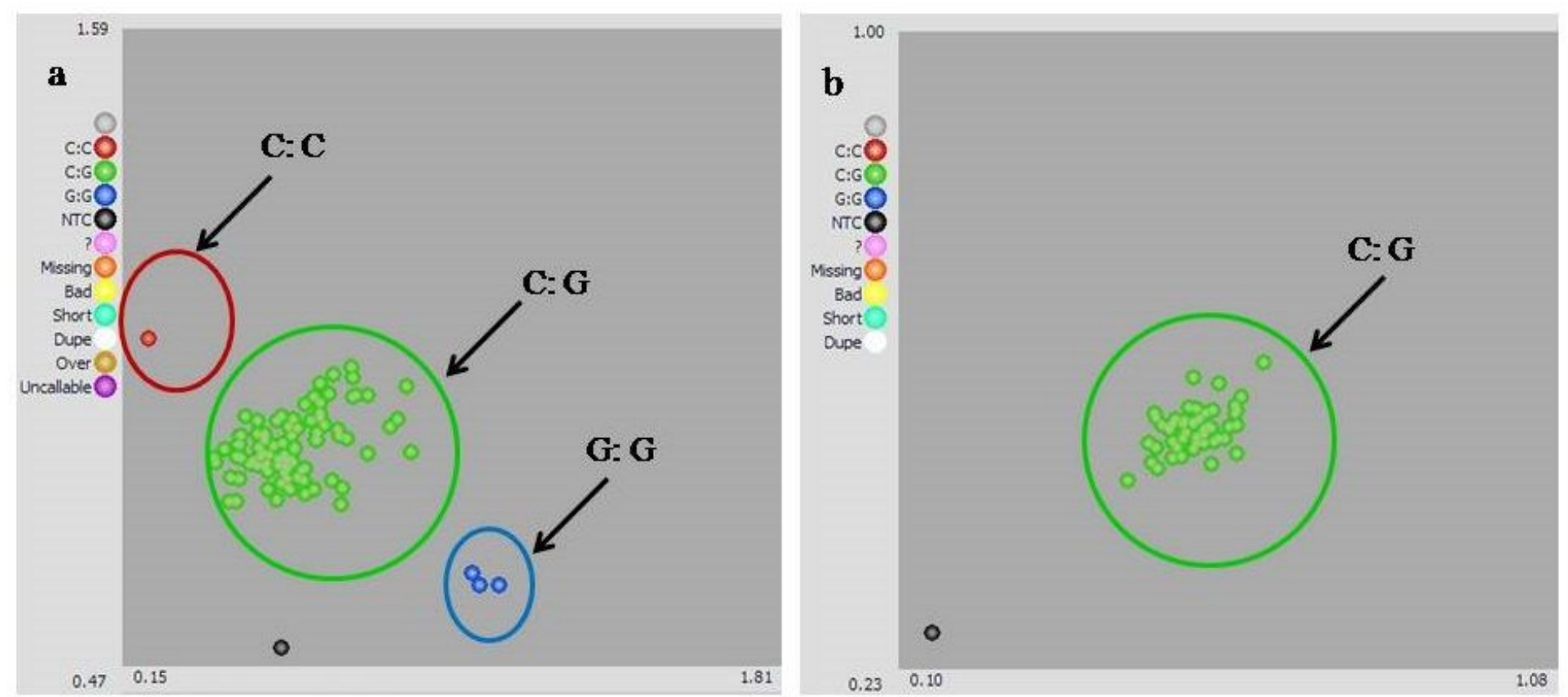

\section{Figure 4}

Test of seed purity for two-line hybrid rice varieties by KASP assay a: KASP assay for Liangyoupeijiu, the genotype of most part of individual plants is heterozygous $\mathrm{C} / \mathrm{G}$ with green signal, and the genotype of three individual plants is homozygous $\mathrm{G} / \mathrm{G}$ with blue signal same with female parent Pei'ai64S, and the genotype of one individual plant is homozygous $\mathrm{C} / \mathrm{C}$ with red signal same with male parent or other conventional plants. b: KASP assay for Hualiangyou1206, the genotype of all individual plants is heterozygous $\mathrm{C} / \mathrm{G}$ with green signal.
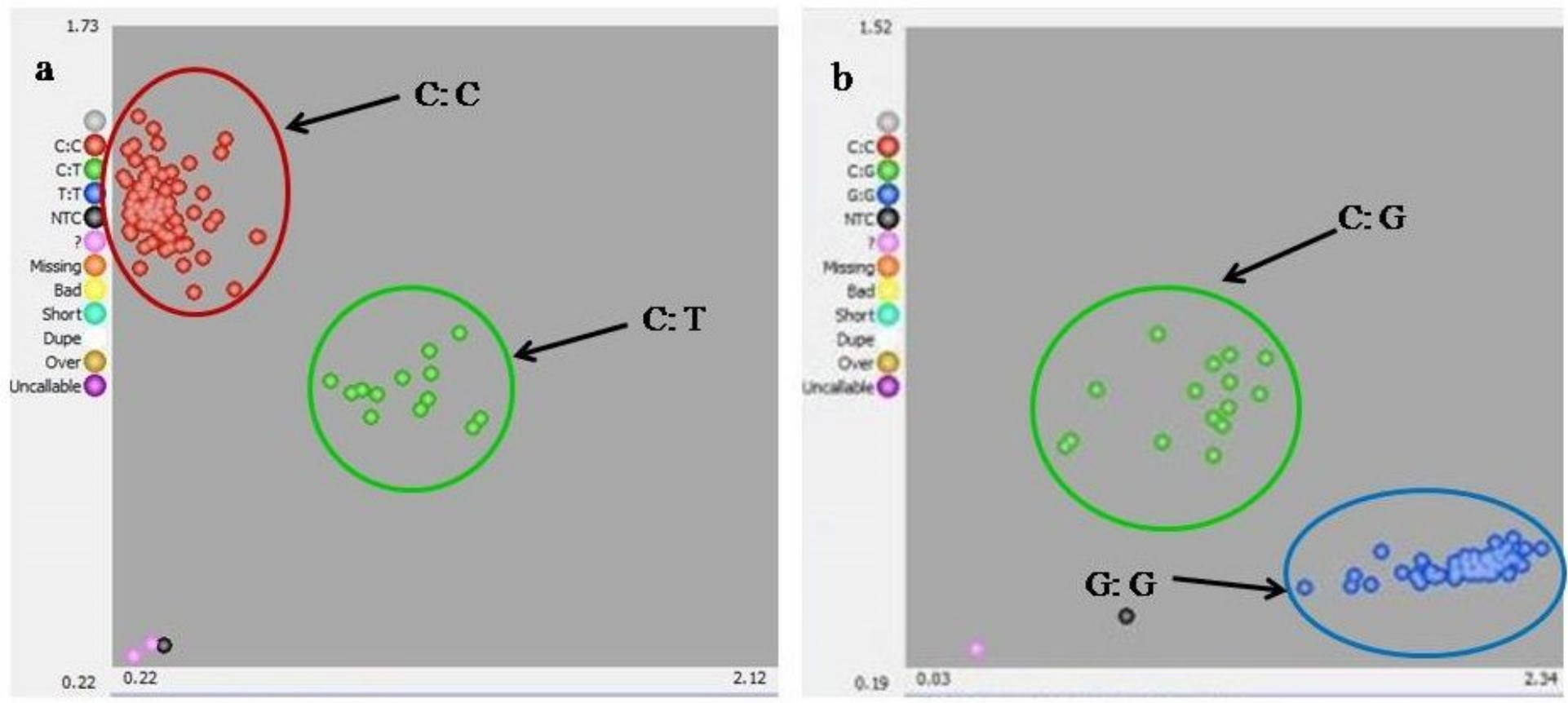

Figure 5 
pyramiding of tms9-1 and p/tms12-1 gene by KASP assay a: KASP assay for tms9-1 gene in F4 population. b: KASP assay for $\mathrm{p} / \mathrm{tms} 12-1$ gene in F4 population. Note: the individual plants with homozygous $\mathrm{G} / \mathrm{G}$ and $\mathrm{C} / \mathrm{C}$ were selected as the TGMS plant pyramiding of tms -1 and $\mathrm{p} / \mathrm{tms} 12-1$ gene.

\section{Supplementary Files}

This is a list of supplementary files associated with this preprint. Click to download.

- Table.pdf 